\title{
Molecular Photonics in Bioelectronic Engineering
}

\author{
Solomon Budnik \\ budnil1@013.net
}

Abstract: New quantum technologies enable to create photonic molecular nanocomputers, biomolecules and cells in the size of a neuron to govern the brain -- a human computer, to distribute information, energy and drugs on molecular level in the human body and cure various deceases.

\section{THEORY}

We deal here with the propagation of matter on molecular level as perceived in my works on physics and astrophysics. (See my papers Quantum Electronics in Quantum Communications, Quantum Photonics, Nanotechnology, Quantum Devices, Theory of Harmonic Propagation of Condensed Matter, Theory of Unified Mater).

Backpath traveling of the particles was predicted in my new quantum field theory. Such motion has nothing to do with the reflection, or wave-particle duality, or backflow effect. It is the backpath energy route for a particle that travels back in spacetime along the trace it left behind, all that due to gravitational pullback to the point of origin after reaching its spin momentum. It's a nuclear expansion and contraction of matter on a nanoscale.

\section{TECHNOLOGY}

Accordingly, superconductivity in my new quantum field model can be combined with molecular supertension due to quantum interference, as the modern development of the Adolph von Baeyer strain theory of the interaction of the tensed ties between atoms and molecules, and the connections between molecular structures and optical properties of the substances.

Accordingly, living cells can be extracted from the human body and polarized to be then reintroduced to the human body for successful medical treatment. This new approach to bioelectronics is termed by me Applied Quantum Photonics.

Term quantum is the quintessence of light, matter, gravity and electromagnetic fields. All said notions mean the creation and existence of life on earth and a new method to cure diseases in a new field of medicine - applied quantum based on my new quantum field theories and book "Beyond Einstein and the Big Bang, NextGen Physics and Astrophysics." My new theory of acoustics is applicable there as well. (See my book "The Absolute Tone, The Secret of Stradivari," combining musical and physical acoustics.

It means that sound, light and e.m. field can be applied externally and internally to the human body to cure various diseases without drugs, surgery, invasive devices and side effects.

For example, heart beat can be restored by external acoustic device to echo normal sounds in the rate of $120 / 80$ to be used instead of cardiostimulators.

Infrared light generated by a certain device via eyes would dissolve spikes between neurons to remedy Alzheimer and prevent strokes, dissolve glaucoma and cataracts, and would direct the harmless heat wave to heat up tumors and destroy them without surgery.

Same concerns sending light signals to the brain via eyes in same electric potential and frequencies in which brain operates when controlling heart, liver, blood pressure and circulation, etc., to restore its full normal control of such processes and cure internal diseases via modulated brain signals. 
Molecular Photonics in Bioelectronic Engineering

In future, atomic signals will be processed via eyes and brain to restore body function on molecular level.

Quantum nanocapsule would be introduced via blood vessels to the body to use cryogenics and UV light to neutralize harmful bacteria and viruses and destroy tumors.

External 3D quantum field would be applied to the body to clean up blood from heavy metals and purify blood instead of dialysis.

\section{CONCLUSION}

Human body functions on internal electric field on acoustic level and those functions will be upgraded in future based on the wave-particle duality known in physics. It means that bionic man could be created in the near future to combine the properties of living and atomic matter.

\section{REFERENCES}

1. Adolph von Baeyer's strain theory

2. Prof. Budnik was involved previously in USA in polymer gel research in medicine. I was the chair, 9th cession at the ICP Prague 2016 18th International Conference on Physics and have now received the speaker invitation for Global Summit on Physics, September 27-28, 2018 in Madrid. My recent paper on quantum fusion has been published by SciFed Journal of Nuclear Science and same concerns my book "Beyond Einstein and the Big Bang, NextGen Physics" to be published soon in Germany.

Citation: Solomon Budnik, “Molecular Photonics in Bioelectronic Engineering”. American Research Journal of Electronics and Communication Engineering; vol 1, no. 1; pp: 21-22.

Copyright (C) Solomon Budnik. This is an open access article distributed under the Creative Commons Attribution License, which permits unrestricted use, distribution, and reproduction in any medium, provided the original work is properly cited. 